\title{
Geometrical and kinematic properties of interfacial waves in horizontal heavy oil-water stratified flow
}

\author{
M. S. de Castro, C. C. Pereira, J. N. dos Santos \\ \& O. M. H. Rodriguez \\ Department of Mechanical Engineering, \\ Engineering School of São Carlos, University of São Paulo, Brazil
}

\begin{abstract}
The oil-water stratified flow pattern is common in the petroleum industry, especially in offshore directional wells and pipelines. Previous studies have shown that the phenomenon of flow pattern transition in stratified flow can be related to the interfacial wave structure (problem of hydrodynamic instability). The study of the wavy stratified flow pattern requires the characterization of the interfacial wave properties, i.e., its nature (kinematic or dynamic), average shape, celerity and geometric properties as a function of holdup and phases' relative velocity. However, the data available in the literature on wavy stratified flow is scanty, especially when oil is viscous. This paper presents geometric and kinematic interfacial wave properties as a function of holdup and relative velocity in the wavy stratified flow. The experimental work was conducted in a glass test line of $12 \mathrm{~m}$ and $0.026 \mathrm{~m}$ i.d., oil (density $828 \mathrm{~kg} / \mathrm{m}^{3}$ and viscosity of $0.3 \mathrm{~Pa} . \mathrm{s}$ at $20^{\circ} \mathrm{C}$ ) and water as the working fluids at horizontal.

Keywords: liquid-liquid flow, oil-water flow, stratified flow pattern, interfacial wave structure.
\end{abstract}

\section{Introduction}

Liquid-liquid flows are present in a wide range of industrial processes; however, studies on such flows are not as common as those on gas-liquid flows. The interest in liquid-liquid flows has recently increased mainly due to the petroleum industry, where oil and water are often transported together for long distances. 
Pressure drop, heat transfer, corrosions and structural vibration are a few examples of topics that depend on the geometrical configuration or flow patterns of the immiscible phases. The stratified flow pattern is present in directional oil wells and pipelines and is characterized by the heavier and lighter phases at the bottom and top part of the pipe, respectively, divided by an interface. The interface in stratified flow can be smooth or wavy. The wavy structure was studied in gas-liquid flow by Bontozoglou and Hanratty [1] and Bontozoglou [2]. One of the findings is that the two-phase friction factor of wavy stratified flow can be about fifty times as high as the friction factor of smooth stratified flow. Li et al. [3] studied the gas-liquid stratified flow pattern and showed that the interfacial waves have significant influence on heat transfer and pressure drop.

An experimental work on liquid-liquid flow patterns can be seen in Trallero et al. [4], where data for horizontal flow were presented including stratified and semi-stratified flow, dispersions and emulsions. Those authors did not differ stratified wavy from stratified with mixing at the interface. Elseth [5] presented a more detailed oil-water horizontal flow pattern classification, dividing Trallero's patterns into several sub-patterns. That author observed the liquid-liquid wavy stratified flow pattern. Alkaya et al. [6] continued the work of Trallero et al. [4], but now introducing the effects of inclination. A wavy stratified flow pattern was reported. All the quoted authors have dealt with relatively low viscosity oils. On the other hand, Bannwart et al. [7] studied very viscous oil-water horizontal flow and reported among others the stratified flow pattern. Interfacial waves were spotted, but details on wave's geometrical properties were not given.

The study of the hydrodynamic stability of liquid-liquid stratified flow and therefore transition to dispersed flow has been carried out by Brauner et al. [8], Trallero [9] and Rodriguez et al. [10]. Although it seems clear that interfacial wave properties are important for the complete understanding of stratified flow, that information is quite scanty. Some data are presented in the paper of AlWahaibi and Angeli [11], but it lacks in details. Therefore, the goal of this work is to investigate geometrical and kinematic properties of interfacial waves in viscous oil-water stratified flow pattern.

In Sections 2 and 3 the experimental setup and procedure are presented. The image processing technique used to measure wave properties is explained in Section 4. In Section 5 data of wavelength, amplitude and celerity of waves are offered. Section 6 presents the mean interfacial wave shape. Finally, the conclusions are drawn in section 7.

\section{Experimental setup}

The hydrophilic-oilphobic glass test line of 26-mm i.d. and 12-m length of the multiphase flow loop of the Thermal-Fluids Engineering Laboratory of the Engineering School of Sao Carlos at the University of Sao Paulo (LETeF) was used to observe different oil-water flow patterns. A by-pass line allowed the usage of the quick-closing-valves technique to measure in-situ volumetric fraction of water and oil. The test section has a transparent box, filled with water, 
used to film the flow. A schematic view of the flow loop and details related to the holdup measurement technique can be seen in Rodriguez et al. [12].

The water used in the experiments has density of $0.988 \mathrm{~g} / \mathrm{cm}^{3}$ and viscosity of $1 \mathrm{cP}$ at temperature $20^{\circ} \mathrm{C}$. The oil used has density of $0.828 \mathrm{~g} / \mathrm{cm}^{3}$ and viscosity of $300 \mathrm{cP}$ at $20^{\circ} \mathrm{C}$. The oil-water interfacial tension was of $0,034 \mathrm{~N} / \mathrm{m}$. The oil-water contact angle with the borosilicate glass was $29^{\circ}$ (hydrophilic-oilphobic). The viscosity was measured with a rheometer Brokfield ${ }^{\mathrm{TM}}$, model LVDV-III+ with rotor SC4-18. The interfacial tension and contact angle were measured with an optical tensiometer of KSVTM, model CAM 200.

\section{Experimental procedure}

The flow was filmed in order to perform the optical measurement of geometrical properties and celerity of the interfacial wave. The film was shot using a high speed video camera (CamRecord 600 from Optronis). The camera was installed on a pedestal attached to the structure. Two xenon lamps were used to illuminate the flow. After reaching steady state the flow was recorded (60 frames/second for 60 seconds) and then a homemade LabView ${ }^{\text {TM }}$ based program was used to extract the properties of the wave from the images.

\section{Data treatment}

The Labview ${ }^{\text {TM }}$ based software was used to identify the wave corresponding to the oil-water interface and predict its amplitude $(\alpha)$, wavelength $(\lambda)$ and celerity (c). Each frame of the film (figure 1) is binarized so that the oil part becomes black and the water part becomes red. Bubbles and other noises are taken off the images. After those steps the interface is captured via a colour contrast technique.

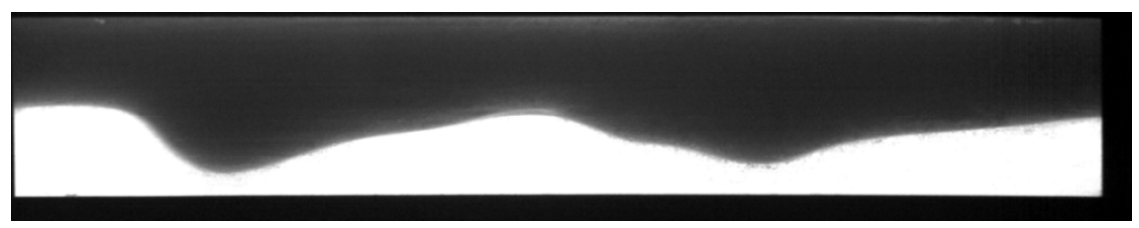

Figure 1: $\quad$ Picture of the observed oil-water wavy stratified flow.

After the identification of the interface the analysis of the wave characteristics depends on the class of wave, related to the internal diameter of the pipeline (D):

Short wave: $\lambda<\mathrm{D}$; it is characterized by a strong corrugated interface, its slope indicates imminence of breaking and its behavior is tridimensional.

Intermediate wave: $\mathrm{D}<\lambda<10 \mathrm{D}$; it is characterized by a clear wavy structure.

Long wave: $\lambda>10 \mathrm{D}$; the interface is almost flat, with small amplitude in relation to the water height.

Using the Fourier transform, the spectrum of waves are represented in the frequency domain. Two cut frequencies were used, one for low-frequency waves and one for high-frequency waves. High frequencies were ignored since they 
were considered noise (ripples). Long waves were not analyzed because they were not entirely visualized in the film section. Hence, short waves and long waves were discarded and only intermediate waves were analyzed.

The wavelength and wave amplitude are calculated using the definition of wave crest as the pixel in the spatial domain where the first derivative is zero, the next pixel has derivative lower than zero and the previous pixel has derivative greater than zero. The wavelength is the length difference between the abscissas (x-coordinate) of two followed crests. Since the interfacial wave is asymmetric, the definition of amplitude is not quite simple. It differs whether it is measured from the crest to the right or left valley (minimum value in the y-coordinate). To avoid this ambiguity, this paper uses the mean between the two calculated amplitudes (from the crest to the right valley and from the crest to the left valley) as the definition of wave amplitude.

The celerity is calculated using cross-correlation between two frames lagged in time (Bendat and Piersol [13]). Figure 2 presents two snap shots used for the measurement of wave celerity.

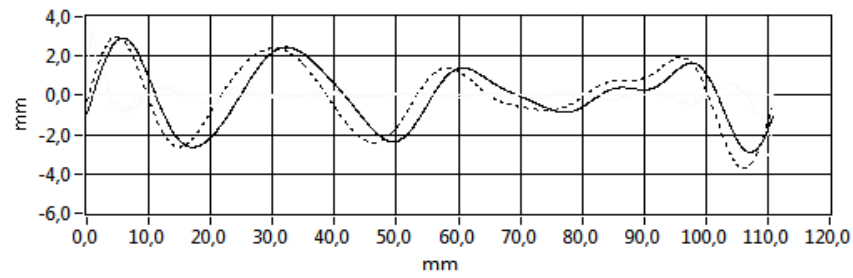

Figure 2: $\quad$ Waves lagged in time.

The PDF (probability density function) of the collected signals was used to analyze the data of amplitude, wavelength and celerity. Around 100 waves were detected by the software in each film.

Two different PDFs of the amplitude data are presented in figure 3, one with oil and water superficial velocities of $0.07 \mathrm{~m} / \mathrm{s}$ and $0.15 \mathrm{~m} / \mathrm{s}$ (figure $3(\mathrm{a})$ ) and another with oil and water superficial velocities of $0.13 \mathrm{~m} / \mathrm{s}$ and $0.15 \mathrm{~m} / \mathrm{s}$ (figure 3(b)), respectively.

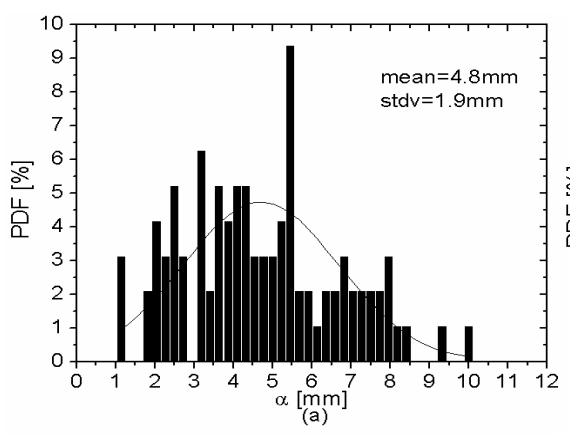

Figure 3: PDF of wave amplitude: (a) $U_{w s}=0.15 \mathrm{~m} / \mathrm{s}$ and $U_{o s}=0.07 \mathrm{~m} / \mathrm{s}$, (b) $U_{w s}=0.15 \mathrm{~m} / \mathrm{s}$ and $U_{o s}=0.13 \mathrm{~m} / \mathrm{s}$.

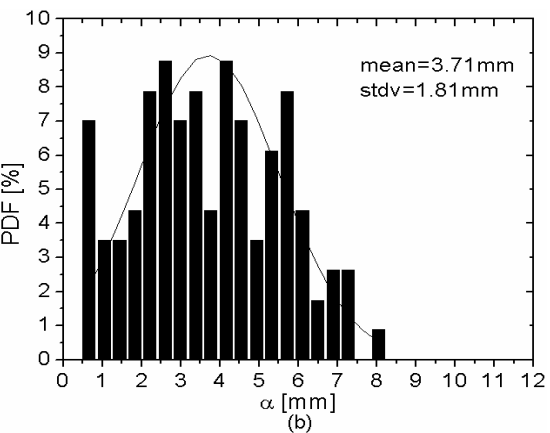

(b) 
The amplitude distribution differs from a Gaussian in both cases. The mean amplitude value is different, but the data have similar distributions (between $1 \mathrm{~mm}$ and $10 \mathrm{~mm}$ ).

Two different PDFs of the wavelength data are presented in figure 4, one with oil and water superficial velocities of $0.15 \mathrm{~m} / \mathrm{s}$ (figure $4(\mathrm{a})$ ) and another with oil and water superficial velocities of $0.15 \mathrm{~m} / \mathrm{s}$ (figure $4(\mathrm{~b})$ ), respectively.

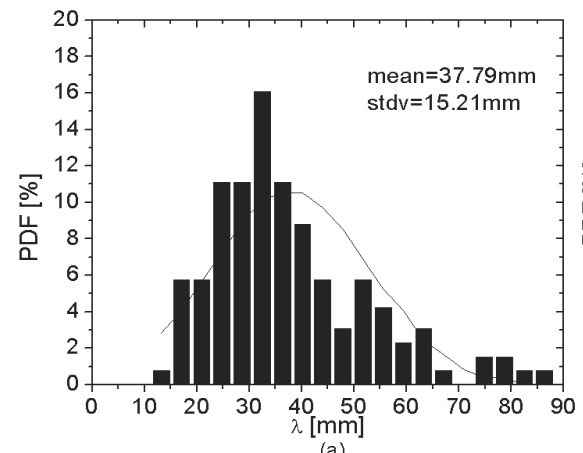

(a)

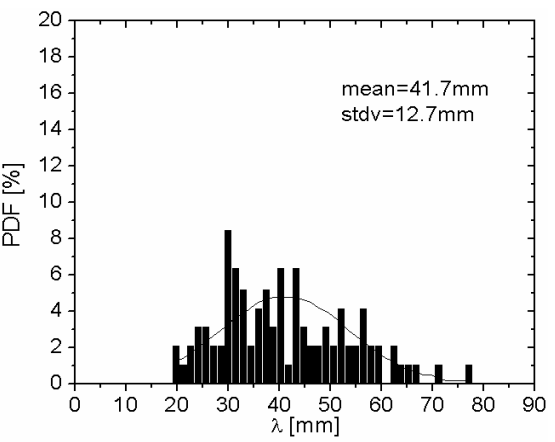

(b)

Figure 4: PDF of wave length: (a) $U_{w s}=U_{o s}=0.15 \mathrm{~m} / \mathrm{s}$, (b) $U_{w s}=0.15 \mathrm{~m} / \mathrm{s}$ and $U_{o s}=0.07 \mathrm{~m} / \mathrm{s}$.

The wavelength PDFs also differ from a Gaussian and have different mean values. While figure 4(a) presents distribution between $10 \mathrm{~mm}$ and $90 \mathrm{~mm}$ with higher concentration between $10 \mathrm{~mm}$ and $40 \mathrm{~mm}$, figure $4(\mathrm{~b})$ presents distribution between $20 \mathrm{~mm}$ and $80 \mathrm{~mm}$, with a more uniform concentration. It is important to notice that there are long wavelength waves, but they represent a small percentage.

Two different PDFs of the wave celerity data are presented in figure 5, with oil and water superficial velocities of $0.05 \mathrm{~m} / \mathrm{s}$ and $0.15 \mathrm{~m} / \mathrm{s}$ (figure $5(\mathrm{a})$ ) and another with oil and water superficial velocities of $0.13 \mathrm{~m} / \mathrm{s}$ and $0.15 \mathrm{~m} / \mathrm{s}$ (figure 5(b)), respectively.

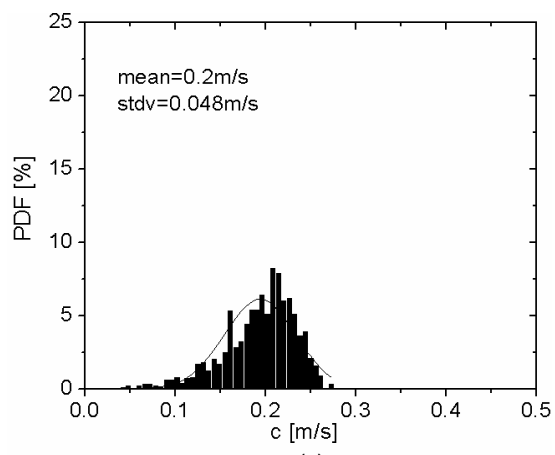

(a)

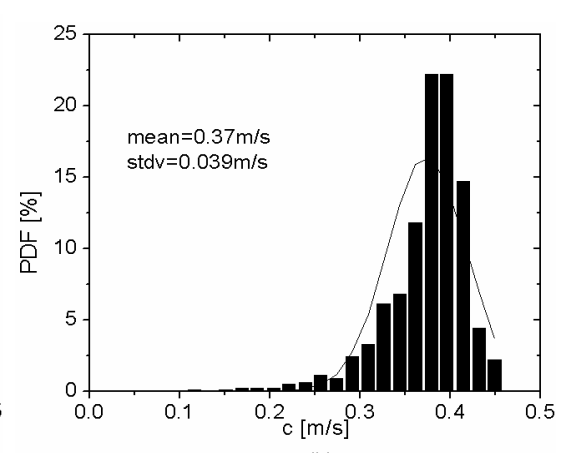

(b)

Figure 5: PDF of wave celerity: (a) $U_{w s}=0.15 \mathrm{~m} / \mathrm{s}$ and $U_{o s}=0.03 \mathrm{~m} / \mathrm{s}$, (b) $U_{w s}=0.15 \mathrm{~m} / \mathrm{s}$ and $U_{o s}=0.13 \mathrm{~m} / \mathrm{s}$. 
One may see that they have different mean values, but similar distribution. Figure 5(a) shows the distribution between $0.05 \mathrm{~m} / \mathrm{s}$ and $0.28 \mathrm{~m} / \mathrm{s}$, whereas figure 5 (b) shows it between 0.1 and $0.45 \mathrm{~m} / \mathrm{s}$. The latter presents a more concentrated distribution around the mean value. Both figures differ from a Gaussian distribution.

\section{Interfacial wave properties}

Table 1 organizes the water and oil superficial velocities at which the experiments were taken.

Table 1: $\quad$ Superficial velocities of oil and water tested.

\begin{tabular}{|c|c|c|}
\hline Experiment & $\begin{array}{c}\text { Superficial velocity of oil } \\
\left(\boldsymbol{U}_{\boldsymbol{~ o s}}\right)[\mathbf{m} / \mathbf{s}]\end{array}$ & $\begin{array}{c}\text { Superficial velocity of water } \\
\left(\boldsymbol{U}_{\boldsymbol{w s}}\right)[\mathbf{m} / \mathbf{s}]\end{array}$ \\
\hline $\mathbf{0 0 1}$ & 0.03 & 0.15 \\
\hline $\mathbf{0 0 2}$ & 0.05 & 0.15 \\
\hline $\mathbf{0 0 3}$ & 0.07 & 0.15 \\
\hline $\mathbf{0 0 4}$ & 0.10 & 0.15 \\
\hline $\mathbf{0 0 5}$ & 0.13 & 0.15 \\
\hline $\mathbf{0 0 6}$ & 0.15 & 0.15 \\
\hline
\end{tabular}

As the wave properties seemed to have relation with relative velocity and holdup the data are presented as function of these variables. Relative velocity is represented by $\mathrm{Vrel}$ (eqn. (1)), where $U_{w}$ and $U_{o}$ are the in situ velocities of oil and water, respectively, and oil holdup is represented by $\varepsilon_{0}$.

$$
\text { Vrel }=U_{w}-U_{o}
$$

\subsection{Interfacial wave length $(\lambda)$}

The normalized mean wavelength $(\lambda / D)$ is presented as a function of the oil holdup and relative velocity in figure $6(\mathrm{a})$ and (b), respectively. One may notice that the amplitude decreases with increasing the oil holdup and relative velocity. The relative velocity is supposed to have a destabilizing effect in the stratified flow (Kelvin-Helmholtz stability criterion, Taitel and Dukler [14]). Short wavelength waves have been related to transition from core-annular flow to dispersed flow (Rodriguez and Bannwart [15], and Rodriguez and Oliemans [16]). Therefore, the highest the Vrel, the highest the destabilizing effect, consequently the shorter the wavelength. On the other hand, high oil holdup implicates in low water volumetric fractions, therefore the effect of gravity in the denser water phase is lower. The gravity has a stabilizing effect, as can be seen in shallow water stability (Whitham [17], Drazin and Reid [18]). So, as the oil holdup increases, the stabilizing effect of gravity decreases and the wavelength becomes shorter. 

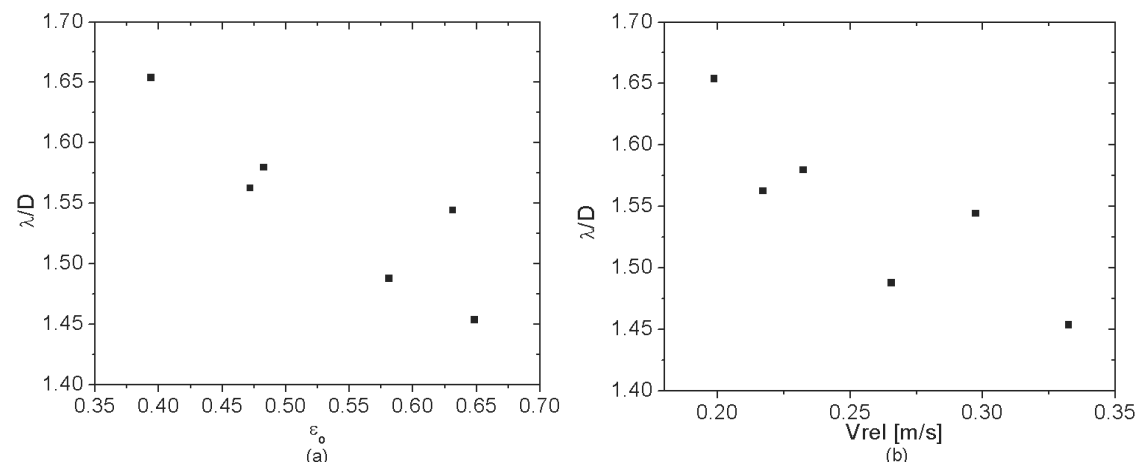

Figure 6: Dimensionless mean wavelength $(\lambda / D)$ as a function of: (a) oil holdup $\left(\varepsilon_{o}\right)$, and (b) relative velocity ( $\mathrm{rel}$ ).

\subsection{Interfacial wave amplitude $(\alpha)$}

The normalized mean wave amplitude $(\alpha / D)$ is presented as a function of the oil holdup and relative velocity in figure 7(a) and (b), respectively. Looking at the graphs of figure 7 one may notice that the amplitude decreases with increasing the oil holdup and relative velocity. It would be expected that for higher oil holdups and relative velocities the flow should become more unstable, which may have been translated into higher wave amplitude. However, it was not seen in the present work. Apparently, shorter waves (refer also to figure 6) have lower amplitudes in the stratified flow investigated in this work.

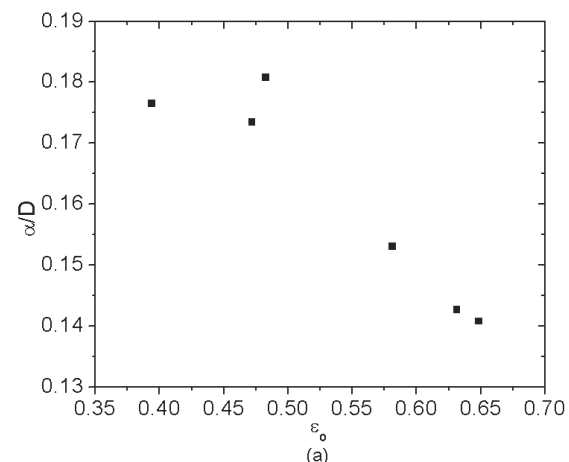

(a)

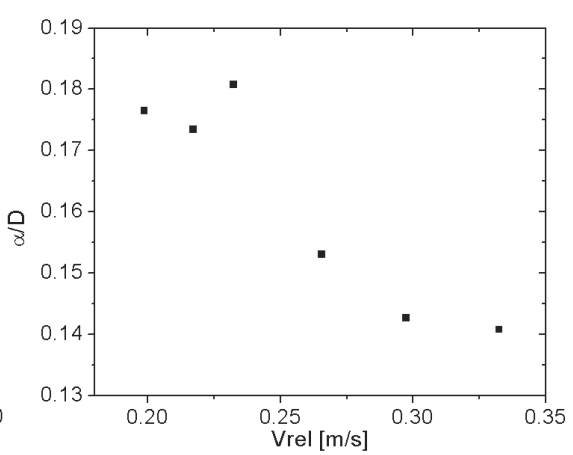

(b)

Figure 7: Dimensionless mean wave amplitude $(\alpha / D)$ as a function of: (a) oil holdup $\left(\varepsilon_{o}\right)$, and (b) relative velocity ( Vrel).

\subsection{Interfacial wave celerity (c)}

The mean interfacial wave celerity is normalized by the mixture velocity $\left(U_{w s}+U_{o s}\right)$ and presented as a function of the oil holdup and relative velocity (figure 8(a) and (b)). The wave celerity increases with increasing both the oil 
holdup and relative velocity. Higher wave celerity would be related to instability of the interfacial wavy structure, as there is a celerity limit for a stable wave in two-phase flow (Wallis [19]).
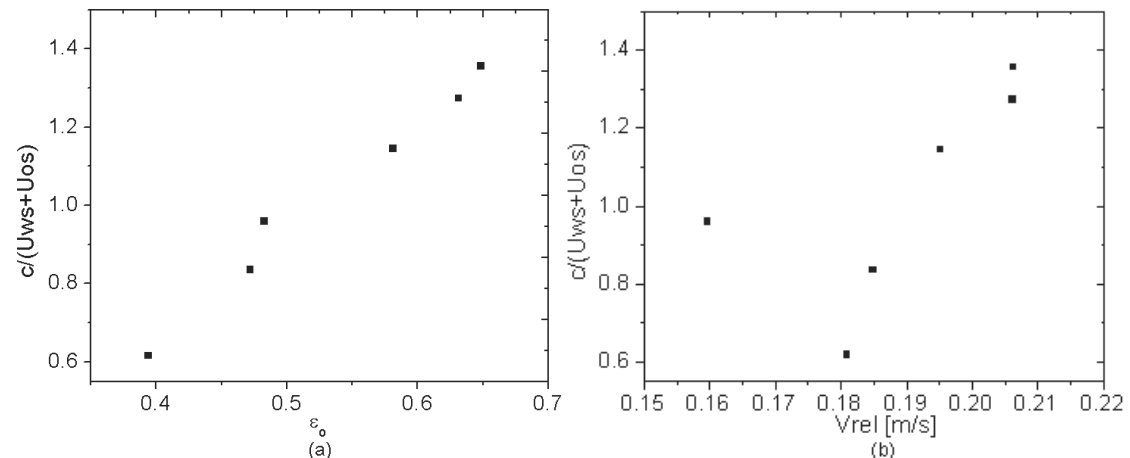

Figure 8: $\quad$ Normalized wave celerity $\left(c /\left(U_{w s}+U_{o s}\right)\right)$ as a function of: (a) oil holdup $\left(\varepsilon_{o}\right)$, and (b) relative velocity ( Vrel).

\subsection{Uncertainties}

This section presents the uncertainties of the collected $\mathrm{d} \alpha_{t} \mathrm{a}$. The values of uncertainties are: $56 \%, 40 \%$ and $40 \%$, respectively, for wave amplitude, wavelength and celerity. The values correspond to 2 times de standard deviation.

The uncertainties of the oil holdup are lower than $3 \%$ and for the relative velocity the uncertainty is of $1.41 \%$.

\section{Mean interfacial wave shape}

The mean interfacial wave shape was found by averaging all the wave shapes captured in the filming for a constant pair of superficial velocities of oil and water. The program divides the waves present in each frame of the film into the previously defined three wave classes, according to the image treatment procedure explained in section 4. Every pair of superficial velocities was related to a number $(i)$ of waves with different amplitudes and wavelengths. Every $\mathrm{x}$ coordinate $(j)$ (abscissa) of the $i$ wave $\left(x_{i j}\right)$ is normalized $\left(x^{*}\right)$ by its own wavelength $\left(\lambda_{i}\right)$, eqn. 2 :

$$
x^{*}=\frac{x_{i j}}{\lambda_{i}}
$$

Every y-coordinate $(j)$ (ordinate) of the $i$ wave $\left(y_{i j}\right)$ is normalized $\left(y^{*}\right)$ by the pipe internal diameter $(D)$, eqn. 3 :

$$
y^{*}=\frac{y_{i j}}{D}
$$


The mean dimensionless wave shape is formed by averaging all the $y^{*}$ points of all waves at every $x^{*}$-coordinate. The mean wave shape is obtained by multiplying the $\mathrm{y}^{*}$ and $\mathrm{x}^{*}$ points of the mean dimensionless wave shape by the pipe diameter and by the mean wave length given by the PDF for each pair of superficial velocities, respectively.

Mean waves shapes are presented in Fig. 9 for the same water superficial velocity, $0.15 \mathrm{~m} / \mathrm{s}$, but for different oil superficial velocities, $0.03 \mathrm{~m} / \mathrm{s}$ and $0.15 \mathrm{~m} / \mathrm{s}$.

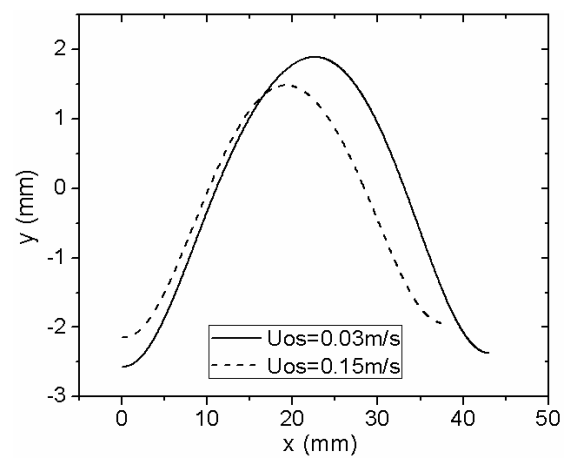

Figure 9: Mean interfacial wave shape for horizontal flow, $U_{w s}=0.15 \mathrm{~m} / \mathrm{s}$.

In Figure 9 the solid line presents data of oil superficial velocity and relative velocity of $0.03 \mathrm{~m} / \mathrm{s}$ and $0.2 \mathrm{~m} / \mathrm{s}$, respectively, and the dashed line presents data of oil superficial velocity and relative velocity of $0.15 \mathrm{~m} / \mathrm{s}$ and $0.33 \mathrm{~m} / \mathrm{s}$, therefore an increase of $33 \%$ in the relative velocity. One may see that the wavelength decreases with the increase of relative velocity. This difference in wave shape might help to explain the stability of stratified flows, i.e., flows with higher relative velocity would tend to be more sensitive to disturbances at the interface or less stable.

\section{Conclusions}

New data of interfacial wave geometrical properties and celerity in viscous oilwater horizontal stratified flow are offered. The wave amplitude, wavelength and wave celerity are dependent on holdup and relative velocity between the water and oil phases. The wavelength tends to decrease with the increase of the relative velocity and with the oil holdup. The celerity tends to increase with the increase of the oil holdup and relative velocity. Both results indicate higher degree of hydrodynamic instability at higher relative velocities. The mean wave shape depends on the relative velocity. The new experimental data and findings can be used for improving pressure drop, holdup and stability models of liquid-liquid stratified flow. 


\section{Acknowledgements}

The authors are gratefull to FAPESP (Fundação de Amparo à Pesquisa do Estado de São Paulo, process 2008/06922-9 and process 2010/03254-5) for the financial support.

\section{References}

[1] Bontozoglou, V. and Hanratty, T.J., Wave Height Estimation In Stratified Gas-Liquid Flows. Aiche Journal, New York, 35 (8), pp. 1346-1350, 1989.

[2] Bontozoglou, V., Weakly Nonlinear Kelvin-Helmholtz Waves Between Fluids Of Finite Depth. International Journal of Multiphase Flow, Oxford, V.17, N.4, P.509-518, July/Aug. 1991.

[3] Li, G.J.; Guo, L.; Chen, X.J., An experimental investigation on the interfacial waves in air-water two-phase flow within horizontal pipelines. Chinese Journal Chemical Engineering, China, 5 (4), pp. 316-324, Dec. 1997.

[4] Trallero, J.L.; Sarica, C.; Brill, J.P., A Study of oil/water flow patterns in horizontal pipes. SPE Production \& Facilities, Richardson, 12 (3), pp.165172, Aug. 1997.

[5] Elseth, G., An experimental study of oil-water flow in horizontal pipes, Ph.D thesis, Porsgrunn: Norwegian University of Science and Technology, 270p, 2001.

[6] Alkaya, B., Jayawardena, S.S., Brill, J.P., Oil Water Flow Patterns in Slightly inclined Pipes. In: Etce/Omae200 Joint Conference: Energy For The News Millennium, 2000, New Orleans. Proceedings. NewYork: Asme. pp. 14-17, 2000.

[7] Bannwart, A.C. et al., Flow patterns in heavy crude oil-water flow. Journal of Energy Resources Technology, 126 (3), pp. 184-189, New York, Sept. 2004.

[8] Brauner, N.; Rovinsky, J.; Maron, D.M., Determination Of The Interface Curvature In Stratified Two-Phase Systems By Energy Considerations. International Journal of Multiphase Flow, Oxford, 22 (6), pp. 1167-1185, 1996.

[9] Trallero, J.L. (1995). Oil-water flow patterns in horizontal pipes. Ph.D. Thesis - The University of Tulsa, Tulsa, 1995.

[10] Rodriguez, O.M.H.; Oliemans, R.V.A.; Mude, R.F. Stability Analysis Of Slightly-Inclined Stratified Oil-Water Flow, Including The Distribution Coefficients And The Cross-Section Curvature. In: Proc. Of the $5^{\text {th }}$ North American Conference On Multiphase Technology, Banff. Proc. Cranfield: Brh Group, pp. 229-245, 2006.

[11] Al-Wahaibi, and P. Angeli, Experimental Studies on Flow Pattern Transitions in Horizontal Oil-Water Flow, Proc. 6th International Conference on Multiphase Flow (ICMF 2007), Leipzig, Germany, July 2007. 
[12] Rodriguez, I. H., Yamaguti, H. K. B., de Castro, M. S., Da Silva, M. J. and Rodriguez, O. M. H., Slip ratio in dispersed viscous oil-water pipe flow, Experimental Thermal and Fluid Science, 35 (1), pp. 11-19, January 2011.

[13] Bendat, J. S. and Piersol, A. G., Random Data: analysis and measurement procedures. John Wiley \& Sons: New York, 2000.

[14] Taitel, Y. and Dukler, A. E., A model for predicting flow regime transitions in horizontal and near horizontal gas-liquid flows. AIChE Journal, 22, pp. 47-55, 1976.

[15] Rodriguez, O.M.H. and Banwart, A. C., Stability Analysis of Core-Annular Flow and Neutral Stability Wave Number, AIChE Journal, 54 (1), January, 2008.

[16] Rodriguez, O. M. H. and Oliemans, R.V. A., Experimental study on oilwater flow in horizontal and slightly inclined pipes, International Journal of Multiphase Flow, 32, pp. 323-343, 2006.

[17] Whitham, G. B. Linear and Nonlinear Waves. New York: John Wiley \& Sons, 1974.

[18] P. G. Drazin, W. H. Reid, Hydrodynamic stability. Cambridge University Press, Cambridge, 1981.

[19] Wallis, G.B., One-Dimensional Two-Phase Flow. MacGraw-Hill, New York, USA, 1969. 